\title{
Vitamin E has Protective Effects on Mice Adrenal Gland which are Exposed to Immobilization Stress
}

FIRAT AŞIR ( $\nabla$ firatasir@gmail.com )

Dicle Üniversitesi https://orcid.org/0000-0002-6384-9146

\section{Yusuf Nergiz}

Dicle University: Dicle Universitesi

\section{Ayşegül Pala}

Dicle University: Dicle Universitesi

\section{Research Article}

Keywords: adrenal gland, immobilization, psychopharmacological tests, Vitamin E

Posted Date: February 16th, 2021

DOI: https://doi.org/10.21203/rs.3.rs-172961/v1

License: (c) (1) This work is licensed under a Creative Commons Attribution 4.0 International License. Read Full License 


\section{Abstract \\ Background}

To investigate the protective effect of vitamin E on mice adrenal glands in immobilization stress and to examine post-stress behavioral changes. Twenty-eight male, 10-week-old, BALB/C mice weighing 30 grams were divided into four groups. Mice were placed in a cage where no movement was allowed 6 hours/day for 7 days for immobilization stress. $0.1 \mathrm{ml}$ saline was administered to the control group and the stress group for 7 days, whereas $30 \mathrm{mg} / \mathrm{kg} /$ day vitamin E was administered orogastrically 1 hour before immobilization stress in the vitamin $E$ and stress + vitamin E group. At the end of the 7th day, all animals were subjected to open field, elevated-plus maze (anxiety) and forced swimming (depression) tests. Left adrenal glands were dissected for routine paraffin tissue protocol. Sections were stained with hematoxylin-eosin and Azan. Malonaldehyde (MDA) levels were also measured in the adrenal tissues.

\section{Results}

Anxiety symptoms were not significant between groups (0.582). Depression level $(p=0.024)$ and MDA values $(p=0.01)$ were the highest in the stress group, which was significantly higher than that of the vitamin E group. In the hematoxylin-eosin sections of the stress group, cortical atrophy, medullary hypertrophy, dilated capillaries, and hemorrhage were observed. Azan staining revealed a thinned capsule, cortical fibrosis, and intense fibrosis in the stress group. Histological structure was protected in the stress + vitamin E group, but dilatation and interstitial fibrosis were also detected.

\section{Conclusion}

Immobilization stress may cause some psychopharmacological, morphometric, and histopathologic changes in mouse adrenal glands, and vitamin E may largely protect the gland from these effects.

\section{Introduction}

Stress is the reaction of the organism to all types of stressogenic stimulants that disrupt its physiological homeostasis. [1] Common stressogenic stimulants used in animal models are heat, coldness, physical restrictions, crowds, noise, immobilization, or isolation. [2] Human beings always experience stressful conditions and the human body copes with these conditions in two ways: i. pumping adrenaline and noradrenaline into the body via the sympathetic nervous system; and ii. activating the hypothalamicpituitary-adrenal (HPA) axis and increasing corticosteroid levels. Both systems fight against stress in the

body. [3] The HPA and sympathoadrenomedullary systems are involved in maintaining the stability of the organism during stress, and the adrenal gland is the final decision-making center in both systems. [4] Vitamin $E$ is a general term for fat-soluble compounds and is found in foods containing fat. It was first discovered by Evans and Bishop in 1922. [5] Vitamin E is classified into two groups by saturated or 
unsaturated side chains. Saturated forms are called tocopherols, and unsaturated form is tocotrienol. Each subgroup has four isoforms: $a, \beta, \gamma, \delta$ tocopherols, and $a, \beta, \gamma, \delta$ tocotrienols. The biologically most active form is a-tocopherols. [6, 7] Vitamin $E$ has been used as an antioxidant for many years. [8, 9] Burton and Traber stated that tocopherols are well recognized for their effective inhibition of lipid peroxidation in foods and living cells.[10] The different forms of tocopherols scavenges free radicals of unsaturated lipids by their antioxidative activity.[11-13]

The aim of this study is to investigate the protective effect of vitamin E against stress-induced changes in mice adrenal glands.

\section{Materials And Methods}

\section{A) Experimental Design}

The study was conducted in accordance with the Basic \& Clinical Pharmacology \& Toxicology policy for experimental and clinical studies [14]. The study was conducted at the Dicle University Animal Research Center. All procedures were approved by the Dicle University Animal Care and Use Local Committee with protocol number 2017/13. Twenty-eight male, 10-week-old, BALB/C mice weighing 30 grams were housed in an air-conditioned room, with 12 -hour light/dark, at $25^{\circ} \mathrm{C}$ temperature and $65-70 \%$ humidity. Mice were assigned to 4 groups (7 mice per group) as the control group, stress group, vitamin E group, and stress + vitamin E group. Using the formulation (30 mg/kg/day dose) [15], 10 grams of $0.1 \mathrm{ml}$ volume of vitamin E (cat\# T325-25G, Sigma, St. Louis, Missouri, USA) was dissolved in olive oil and administered to mice through the orogastric route for 7 days. Only $0.1 \mathrm{ml}$ of saline was given daily through the orogastric route to the control and stress groups. The weights of all mice were recorded before the experiments.

The mice were placed in a space as small as their size to prevent their movement. This procedure was performed 6 hours/day for 7 days [16]. In the stress + vitamin E group, the medication was given one hour before the experiment protocol.

\section{B) Psychopharmacological Tests}

At the end of the 7th day, all groups were subjected to open field [17], elevated-plus maze [18] and forced swimming tests [19] to measure anxiety and depression levels in mice.

\section{Open field test}

This test was performed to assess locomotor activity and anxiety-like behavior in mice. Briefly, a $40 \times 40 \times 20 \mathrm{~cm}$, upside open, white wooden box with black walls was used. Mice were initially placed in the center and spontaneous ambulatory locomotion of each mouse was recorded with EthoVision ${ }^{\circledR}$ XT 11.0 (Noldus Inf. Tech, Netherlands) for 5 minutes. Time spent in the center (index of anxiety) and total movement distance were recorded. 


\section{Elevated-Plus maze}

The mice were observed with the EthoVision ${ }^{\circledR}$ digital software for 5 minutes in a maze $50 \mathrm{~cm}$ high from ground. The maze had four $75 \mathrm{~cm}$ long arms, two open and two closed ends. The mice were placed in the intersection of ends and their movements toward the closed/open ends were recorded to evaluate their anxiety and motor function.

\section{Forced Swimming Test}

This test was performed to assess the depression levels of mice. The mice were marked with a digital camera and swam in a cylinder with a radius of $20 \mathrm{~cm}$, a height of $50 \mathrm{~cm}$, and $2 / 3$ of water $\left(25^{\circ} \mathrm{C}\right)$ for 6 minutes. To calculate depression level, the motion and motionless time periods in animal behaviors were recorded for the last 4 minutes by the EthoVision ${ }^{\circledR}$ XT 11.0 (Noldus Inf. Tech, Netherlands) software.

\section{C) Malonaldehyde (MDA) Level Analysis}

At the end of the experimental protocols, the mice were weighed and then sacrificed by cervical decapitation under ether anesthesia. All adrenal gland tissues were dissected and stored in $-80^{\circ} \mathrm{C}$ for Malonaldehyde (MDA) analysis.

\section{D) Morphometric Measurements}

Total body weights of all mice in all groups were recorded before and after the experiment. The diameters of the capsules and cortices were measured by ZEN lite for Windows (ZEISS Microscopy, Carl Zeiss Promenade, Germany).

\section{E) Tissue Processing for Histopathology}

The left adrenal glands were also dissected and fixed in $10 \%$ formalin solution. Routine paraffin wax embedding procedure was followed and $5-\mu \mathrm{m}$ sections were stained with hematoxylin-eosin (H-E) (cat \# W01030708, Bioptica, Milano, Italy) and Mallory Azan (cat \# 04-020802, Bioptica, Milano, Italy). The slides were evaluated under Zeiss Axio imager A2 (Carl Zeiss Microscopy, LLC, USA) light microscope and photomicrographed.

\section{F) Statistical Analysis}

All data were analyzed using IBM SPSS Statistics 25 (SPSS Co., Chicago, IL, USA). Results were shown in mean \pm standard deviation (SD). Comparisons between groups were calculated by one-way analysis of variance (ANOVA) and then further analyzed by post-Hoc Tukey test. A $p$ value $<0.05$ was considered as statistically significant. All raw data of psychopharmacological test, morphometric measurements and MDA values was supplied as Supplemental data file.

\section{Results}

\section{Psychopharmacological Findings}




\section{Open Field Test}

The difference between groups was not statistically significant in terms of total covered distance $(p=$ 0.295 ) and time spent in the center ( $p=0.582$, index of anxiety) (Fig. 1$)$.

\section{Elevated Plus Maze}

When compared with the control group, a decrease in the time consumed in the open ends was observed in the stress group ( $p=0.023$, index of anxiety). Time spent in the open ends was shown in Fig. 2 for each group.

\section{Forced Swimming Test}

Regarding immobilized time of mice passed in the forced swimming test is shown in Fig. 3. The highest immobilized time was in the stress + vitamin E group. The decreased time in the vitamin E group was statistically significant compared to the stress group. The immobilized time in the stress group was statistically higher than in that of the control group $(p=0.04)$.

\section{C) MDA Analysis}

Tissue MDA value of the stress group was significantly higher than that of the control group. The vitamin $E$ and the stress + vitamin E showed lower MDA value than stress group and the results were statistically significant ( $p=0.01$ and $p=0.034)$ (Fig. 4).

\section{D) Morphometric Findings}

All groups showed no statistically significant changes in total body weight before and after the experiment $(p>0.05)$ (Fig. 5).

\section{E) Histopathological Findings}

Capsule thickness was significantly lower in the stress group compared to the vitamin E and control groups ( $p=0.016$ and $p=0.048$, respectively). However, the difference between vitamin $E$ and stress groups was relatively less than that of the vitamin $E$ and control groups. There was no significant difference between the stress group and the stress + vitamin E group ( $p>0.05)$ (Fig. 6a).

Cortex thickness of both vitamin $E$ and control groups was significantly higher than that of the stress group $(p=0.003$ and $p=0.016$, respectively). However, cortex thickness between stress and stress + vitamin E groups was not significant $(p>0.05)$ (Fig. 6b).

\section{Hematoxylin-Eosin staining}


The adrenal gland was histologically normal with a fibrous capsule in the control group. The zones of glomerulosa, fasciculata, and reticularis were evident. (Fig. 7a). In sections of the stress group, cortical atrophy and medullary hypertrophy were observed. The size of all three cortical zones reduced, but the zone most atrophied was the zona glomerulosa. Vascular dilatation and hemorrhage were seen in the medulla (Fig. 7b). Adrenal sections of the vitamin E group showed normal histological structure with the capsule, cortical layers, and medulla. The fibrous capsule, cortex, and medulla were histologically similar to the control group (Fig. 7c). In the stress + vitamin E group, histopathological findings (size of cortex, hemorrhage, vascular dilation, apoptosis) were mostly recovered compared to the stress group. The appearance of zones of glomerulosa, fasciculata, and reticularis and medulla seemed closer to that of the control group (Fig. 7d).

\section{AZAN staining}

In the sections of the control group stained with AZAN, the adrenal gland was histologically normal. Capsule was normal, and zona glomerulosa beneath the capsule was observed with dense nuclei and mitotic activities (Fig. 8a). In the sections of the stress group, the capsule diameter decreased, and the medulla size increased. Some regions of medulla had minimal interstitial fibrosis, while dense fibrosis was observed in the corticomedullary region (Fig. 8b). In the sections of the vitamin E group, normal adrenal gland histology was observed. Capsule, cortical zones, and medulla were structurally similar to the control group (Fig. 8c). In the stress + vitamin E group, decreased interstitial fibrosis and vascular dilatation were observed. The histological structure of the adrenal cortex and medulla were similar to the control group (Fig. 8d).

\section{Discussion}

In this study, the effects of vitamin E on the adrenal gland were investigated in the immobilization stress model. Mice were subjected to immobilization stress six hours a day for one week. Our results revealed that stress-induced structural changes such as cortical atrophy, medullary hypertrophy, dilated capillaries, fibrosis, and hemorrhage were observed in the adrenal gland, confirming the findings of previous studies. $[4,20,21]$ Moreover, we have demonstrated that vitamin $E$ has a protective effect on these stress-induced changes.

Stress is a process that plays an active role in the pathogenesis of various diseases in rodents by deteriorating the physical, biochemical, and psychological parameters. [22] A study showed that stress has the potential to increase symptoms of anxiety and depression. [23] In this study, immobilization stress also caused severe anxiety-like behavior and depression as compared with unstressed animals. Experimental studies demonstrated that vitamin E has antidepressant-like effects. [24, 25] The depression level of the mice in the stress group increased significantly in the forced swimming test, while the depression level of the vitamin E group was decreased (Figs. 1,2 and 3). 
Exposure to stress may elevate the production of free radicals and impair the antioxidant defense system, leading to oxidative damage and imbalance between oxidant and antioxidant factors. [26-28] We measured MDA (a biomarker of lipid peroxidation) levels, which was the highest in the stress group. MDA levels in the vitamin E treated groups were significantly lower than that of the stress group (Fig. 4). These results confirmed that vitamin $\mathrm{E}$ has antioxidant effects that can protect the adrenal gland tissue from stress-induced oxidative stress.

Exposure to immobilization stress also causes histopathological changes in the stomach, intestine, testis, and adrenal gland in male rats. [29-31] Morphometric measurements revealed that capsule and cortex thickness significantly decreased in the stress group (Fig. 3). Studies have shown that the zona glomerulosa is the major zone of mitosis and that the zona reticularis is the region with most cell deaths. [32-34] The histochemical observations in the present work showed that the control and vitamin $\mathrm{E}$ groups indicated similar histological appearances (Fig. 7a, c). Cortical atrophy was observed in the stress and stress + vitamin E groups. This was because of the balance between mitosis and cell death. In our case the balance was in favor of cell death. However, atrophy in the stress + vitamin E group was lower than in the stress group, suggesting vitamin $E$ induces cortical cells to drive mitosis.[33] Vascular dilatation, congestion, and hemorrhage were also seen in this group. Generally, the stress + vitamin $\mathrm{E}$ group showed histology similar to the control group (Fig. 7b,d).

El-Refaiy [35] and Rai et al [36,37] showed that exposure to stress increased fibroblasts and collagen fiber synthesis, leading to the thickening of the testis basement membrane. Furthermore, increased fibrosis in the colon mucosa of rats exposed to stress due to collagen synthesis has also been documented. [38] El-Desouki et al. [25] stated that immobilized stress ultrastructurally caused elevated levels of collagen fibers in the rat adrenal cortex. In the AZAN-stained sections, the control group and the vitamin E group showed no fibrosis (Fig. 8a, C). Interstitial fibrosis through the adrenal cortex was seen in the stress group, but dense fibrosis was observed in the corticomedullary region. Excessive vascular dilatation, congestion, and hemorrhage were also observed in the stress group. In the stress + vitamin $\mathrm{E}$ group, those symptoms were mostly recovered, but signs of fibrosis were still evident (Fig. 8b, d).

In conclusion, immobilization stress may be responsible for the psychopharmacological, morphometric, and histochemical changes in the immobilization stress-induced mouse adrenal gland, and vitamin $\mathrm{E}$ may be protective against these alterations.

\section{Declarations}

\section{Ethics approval and consent to participate}

Ethics approval was obtained by Dicle University Animal Care and Use Local Committee with protocol number 2017/13.

\section{Consent to participate}


Not Applicable

\section{Consent for publication}

Not Applicable

\section{Availability of data and materials}

All data generated or analyzed during this study are included in this published article and its

Supplementary Files.

\section{Competing interests}

All authors declare no conflict of interest. The authors have nothing to disclose.

\section{Funding}

This research received no specific grant from any funding agency in the public, commercial, or not-forprofit sectors.

\section{Authors' contributions}

"FA, YN and AP conceptualized and designed the experiments, performed the histological examination of the adrenal gland and psychopharmacological tests, and were equally contributor in writing the manuscript. All authors read and approved the final manuscript. The authors declare that all data were generated in-house and that no paper mill was used.

\section{Acknowledgements}

We kindly thank to Dr. Emre UYAR for statistical analysis.

\section{References}

1. Chrousos GP, Gold PW (1992) The concepts of stress and stress system disorders. Overview of physical and behavioral homeostasis. JAMA 267(9):1244-1252

2. Djordjevic J, Cvijic G, Davidovic V (2003) Different activation of ACTH and corticosterone release in response to various stressors in rats. Physiol Res 52(1):67-72

3. Axelrod J, Reisine TD (1984) Stress hormones: their interaction and regulation. Science 224(4648):452-459

4. Petrovic-Kosanovic D et al (2012) Effect of acute heat stress on rat adrenal cortex - a morphological and ultrastructural study. Central European Journal of Biology 7(4):611-619

5. Niki E, Traber MG (2012) A history of vitamin E. Ann Nutr Metab 61(3):207-212 
6. Mitchell AD, Benevenga NJ (1978) The role of transamination in methionine oxidation in the rat. J Nutr 108(1):67-78

7. Wang X, Quinn PJ (1999) Vitamin E and its function in membranes. Prog Lipid Res 38(4):309-336

8. Tappel AL (1980) Vitamin E and selenium protection from in vivo lipid peroxidation. Ann N Y Acad Sci 355:18-31

9. Burton GW, Joyce A, Ingold KU (1982) First proof that vitamin E is major lipid-soluble, chain-breaking antioxidant in human blood plasma. Lancet 2(8293):327

10. Burton GW, Traber MG (1990) Vitamin E: antioxidant activity, biokinetics, and bioavailability. Annu Rev Nutr 10:357-382

11. Burton GW, Ingold KU (1981) Autoxidation of biological molecules. 1. Antioxidant activity of vitamin E and related chain-breaking phenolic antioxidants in vitro. J Am Chem Soc 103(21):6472-6477

12. Niki E et al (1984) Inhibition of oxidation of methyl linoleate in solution by vitamin $E$ and vitamin $C . J$ Biol Chem 259(7):4177-4182

13. van Acker SA, Koymans LM, Bast A (1993) Molecular pharmacology of vitamin E: structural aspects of antioxidant activity. Free Radic Biol Med 15(3):311-328

14. Tveden-Nyborg P, Bergmann TK, Lykkesfeldt J, Basic \& Clinical Pharmacology \& Toxicology Policy for Experimental and Clinical studies. Basic Clin Pharmacol Toxicol (2018) 123(3): p. 233-235

15. Yargicoglu $P$ et al (2003) The effect of vitamin E on stress-induced changes in visual evoked potentials (VEPs) in rats exposed to different experimental stress models. Acta Ophthalmol Scand 81(2):181-187

16. Inoue T et al (1993) Effects of single and repeated immobilization stress on corticotropin-releasing factor concentrations in discrete rat brain regions. Prog Neuropsychopharmacol Biol Psychiatry 17(1):161-170

17. Carli M, Prontera C, Samanin R (1989) Effect of 5-HT1A agonists on stress-induced deficit in open field locomotor activity of rats: evidence that this model identifies anxiolytic-like activity. Neuropharmacology 28(5):471-476

18. Pellow $S$ et al (1985) Validation of open:closed arm entries in an elevated plus-maze as a measure of anxiety in the rat. J Neurosci Methods 14(3):149-167

19. Burgin R, Weizman R, Gavish M (1996) Repeated swim stress and peripheral-type benzodiazepine receptors. Neuropsychobiology 33(1):28-31

20. Adzic $M$ et al., Effect of different types of stress on adrenal gland parameters and adrenal hormones in the blood serum of male Wistar rats. Archives of Biological Sciences, 2009. 61

21. Ulrich-Lai YM et al (2006) Chronic stress induces adrenal hyperplasia and hypertrophy in a subregion-specific manner. Am J Physiol Endocrinol Metab 291(5):E965-E973

22. Pablos Ro, Sarmiento M, Espinosa-Oliva A, Creating a Rat Model of Chronic Variate Stress. BIOPROTOCOL, 2014. 4 
23. Chen LJ et al (2014) The effects of early-life predator stress on anxiety- and depression-like behaviors of adult rats. Neural Plast 2014:163908

24. Lobato KR et al (2010) alpha-Tocopherol administration produces an antidepressant-like effect in predictive animal models of depression. Behav Brain Res 209(2):249-259

25. De Marchis GM et al (2006) Vitamin E reduces antidepressant-related beta-adrenoceptor downregulation in cultured cells. Comparable effects on St. John's wort and tricyclic antidepressant treatment. Planta Med 72(15):1436-1437

26. Bagchi $D$ et al (1999) Acute and chronic stress-induced oxidative gastrointestinal mucosal injury in rats and protection by bismuth subsalicylate. Mol Cell Biochem 196(1-2):109-116

27. Rai J, Srivastava PS (2004) RK, Testosterone hormone level in albino rats following restraint stress of long duration. J Anat Soc 53(1):17-19

28. Ganesan B, Anandan R, Lakshmanan PT (2011) Studies on the protective effects of betaine against oxidative damage during experimentally induced restraint stress in Wistar albino rats. Cell Stress Chaperones 16(6):641-652

29. Gabry KE et al (2002) Marked suppression of gastric ulcerogenesis and intestinal responses to stress by a novel class of drugs. Mol Psychiatry 7(5):474-483

30. Desoky $\mathrm{N}$ et al (2012) Histological, histochemical and immunohistochemical studies on the cardiac muscle of albino rat under immobilization stress and the possible curative role of diazepam. Egypt $\mathrm{J}$ Exp Biol(Zool) 8:273-285

31. El-Desouki NI, Abdel-Azeem E-RA, El-Baely H (2011) MA., Histological and ultrasructural studies of the effect of immobilization stress on the adrenal cortex of albino rat and the ameliorative role of diazepam. Egypt Ger Soc Zool 62:25-45

32. Wright N, Voncina D (1977) Studies on the postnatal growth of the rat adrenal cortex. J Anat 123(Pt 1):147-156

33. Stachowiak A, Nussdorfer GG, Malendowicz LK (1990) Proliferation and distribution of adrenocortical cells in the gland of ACTH- or dexamethasone-treated rats. Histol Histopathol $5(1): 25-29$

34. Miyamoto $\mathrm{H}$ et al (2000) Daily regeneration of rat adrenocortical cells: circadian and zonal variations in cytogenesis. Endocr Res 26(4):899-904

35. Al E-R (2010) Histological and histochemical studies on the testes of albino rat under immobilization stress and the possible curat ive role of diazepam. J Egypt Ger Soc Zool 60(C):1-22

36. Rai J, Srivastava PS (2003) RK., Effect of immobilization stress on spermatogenesis of albino rats. J Anat Soc India 52(1):55-57

37. Rai J, Srivastava PS (2004) RK., Testosterone hormone level in albino rats following restraint stress of long duration. J Anat Soc India 53(1):17-19

38. El-Derieny EA (2006) M.A., Histological study on the effect of stress on the colonic mucosa of adult male albino rat and possible role of mast cells. Egypt J Histol 29(2):315-326 
Figures

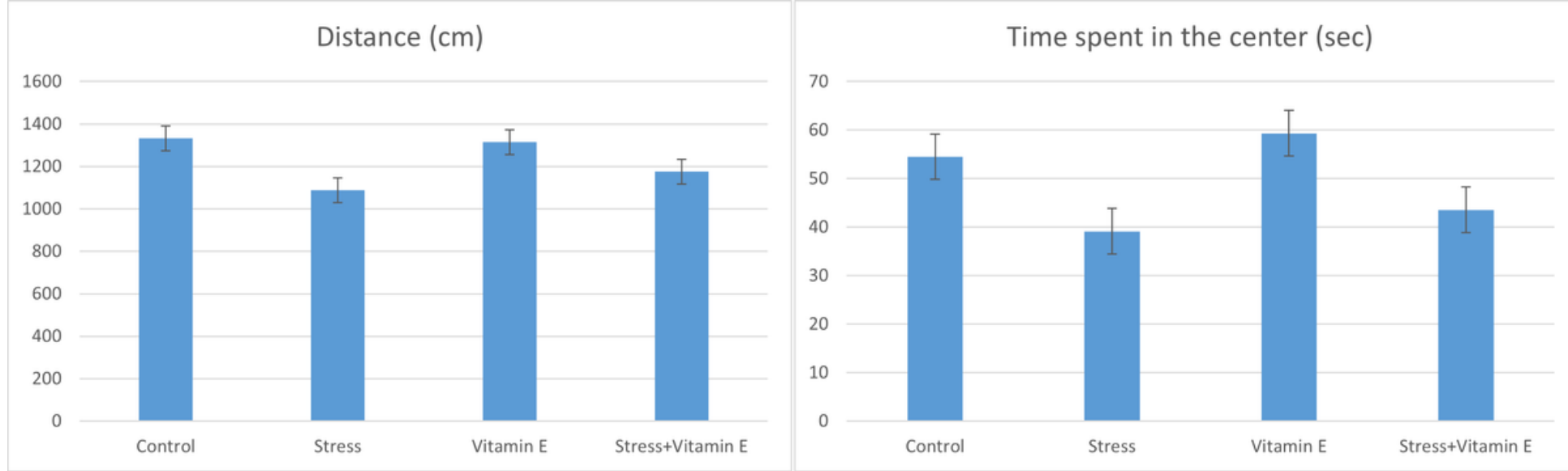

Figure 1

shows open field test parameters (distance covered and time spent in the center) for all groups.

\section{Time spent in the open ends (\%)}

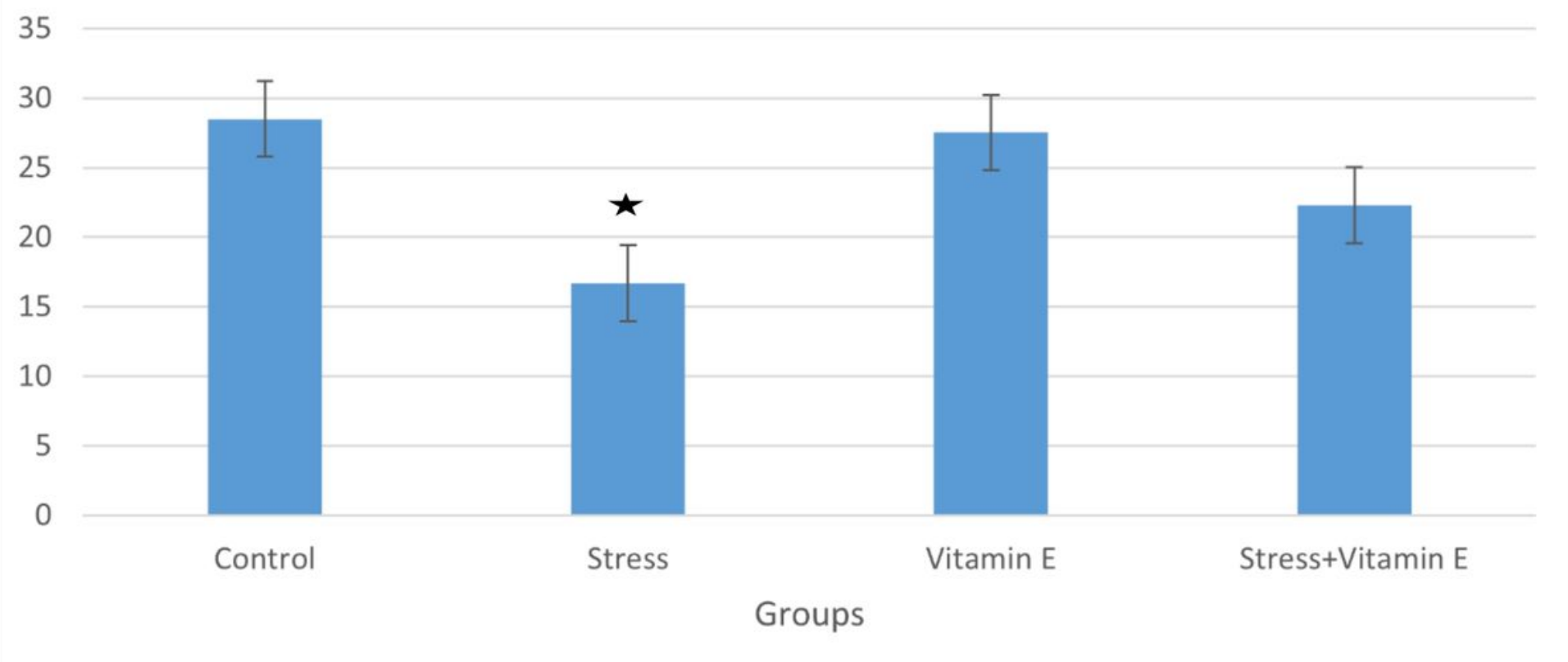

Figure 2

shows elevated plus maze results (time spent in the open ends) for all groups. * shows significance of the stress vs control group. 


\section{Immobilized Time (sec)}

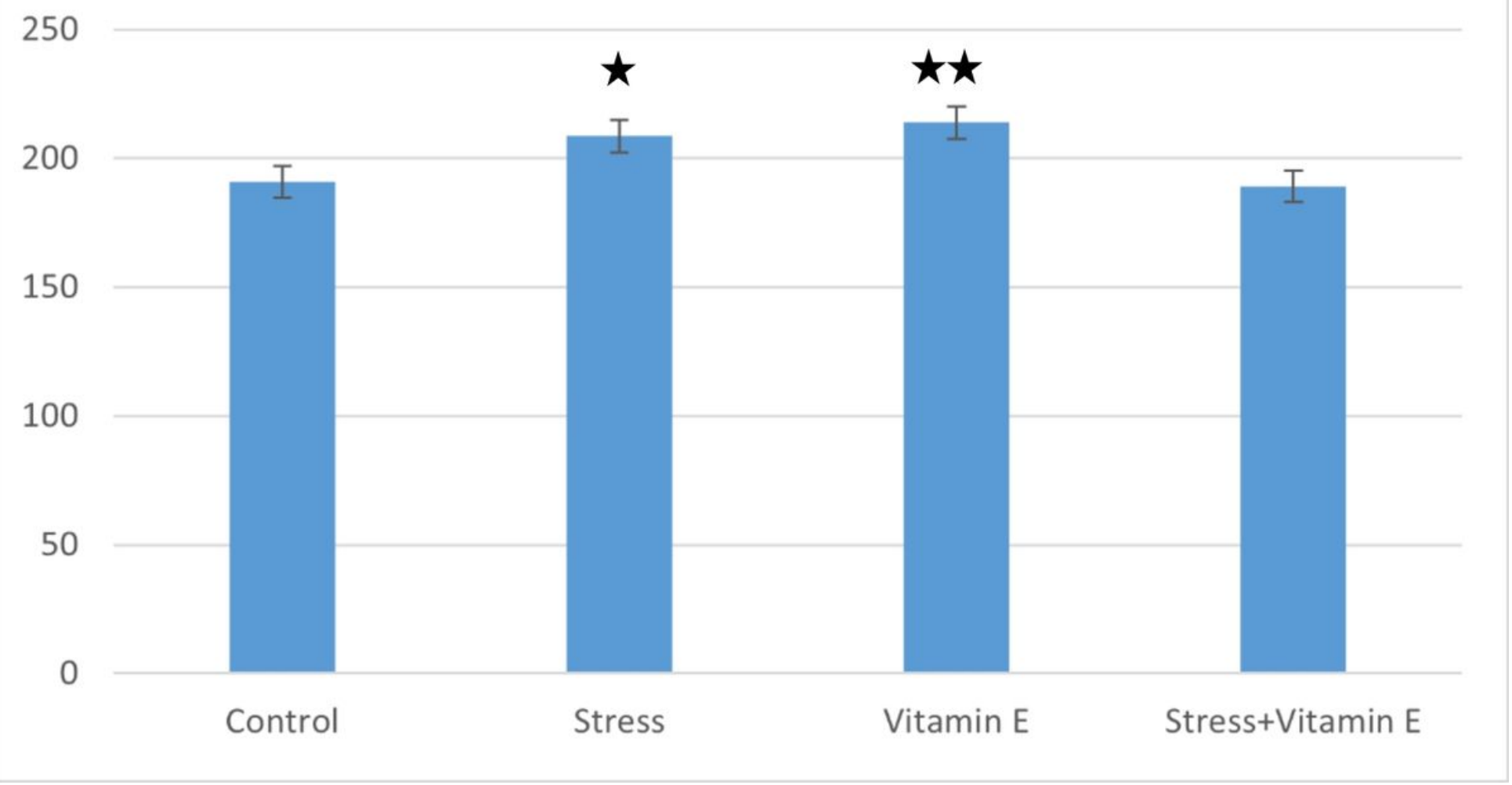

\section{Figure 3}

shows the immobilized time passed in the forced swimming test for all groups. * shows significance of stress vs. control group, ** vitamin E vs. stress groups. 


\section{Tissue MDA Value}

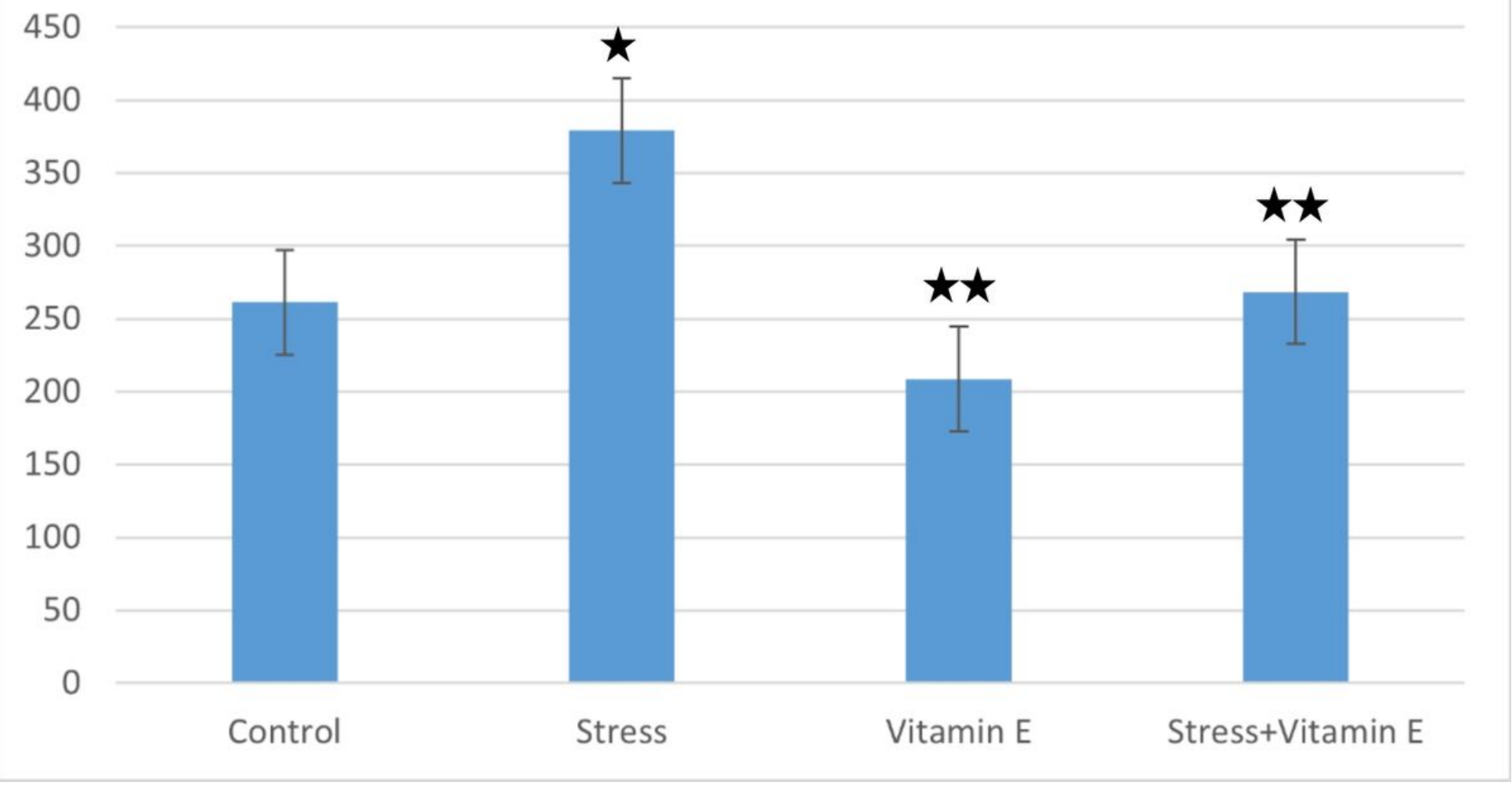

Figure 4

shows the tissue MDA values of all groups. ${ }^{*}$ shows significance of the stress vs control group, ${ }^{\star *}$ Vitamin E treated groups vs stress groups.

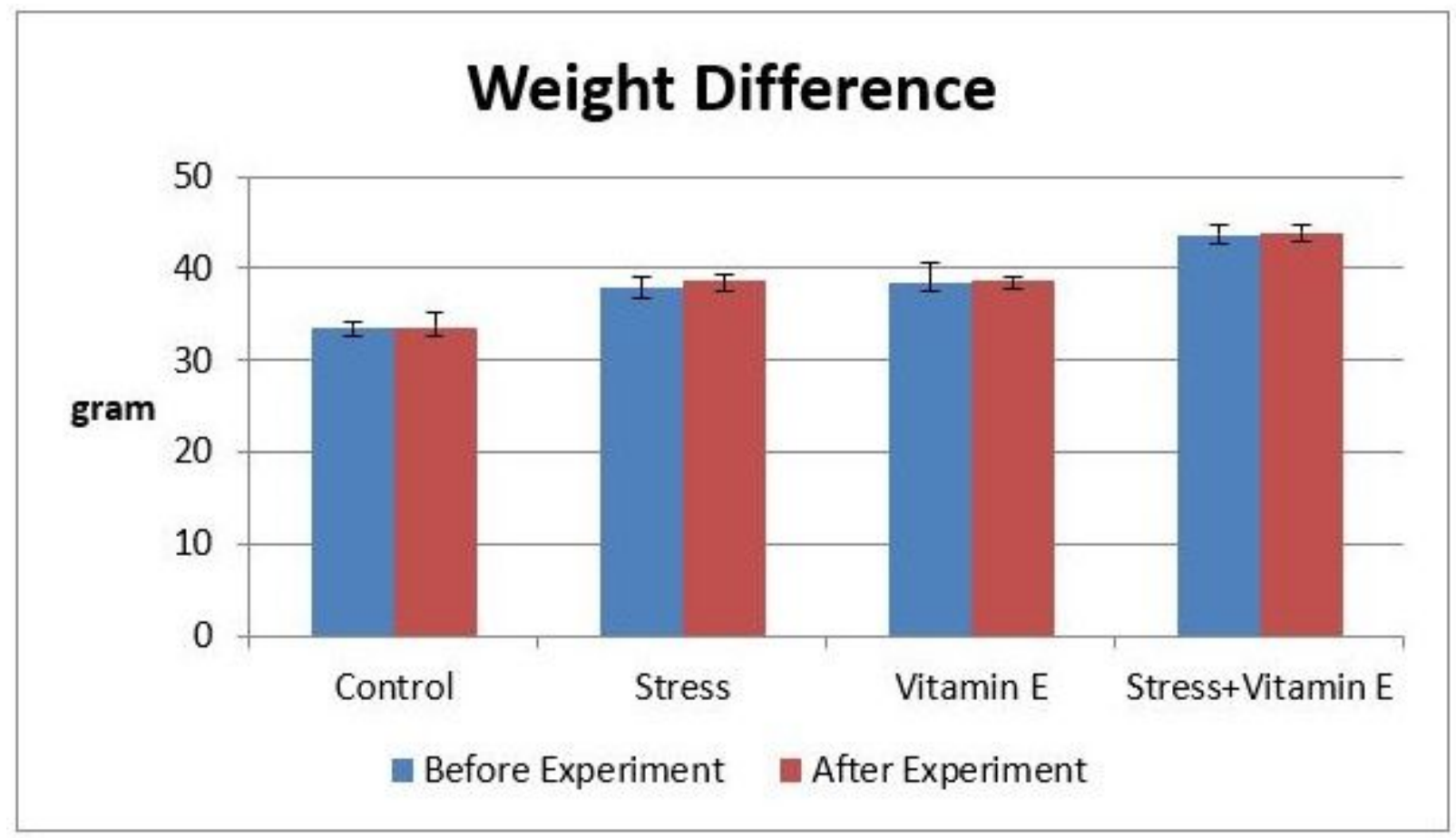

Figure 5 
Difference in total body weights before and after the experimental protocols for all groups.

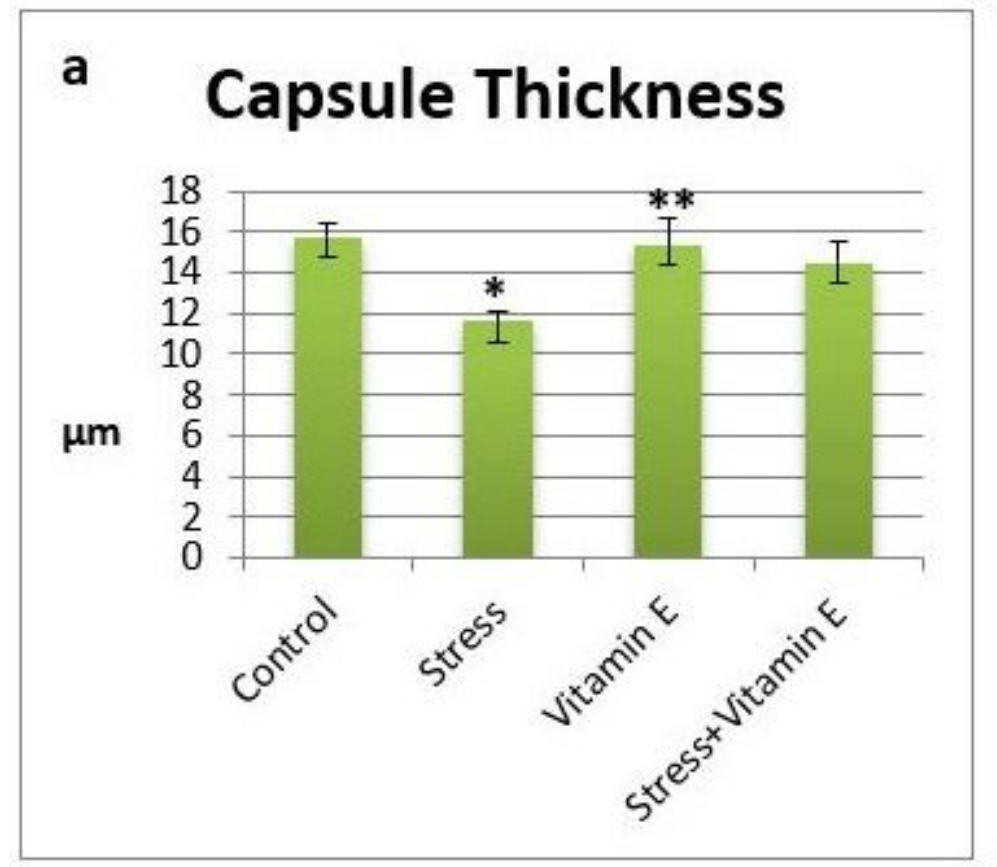

\section{b Cortex Thickness}

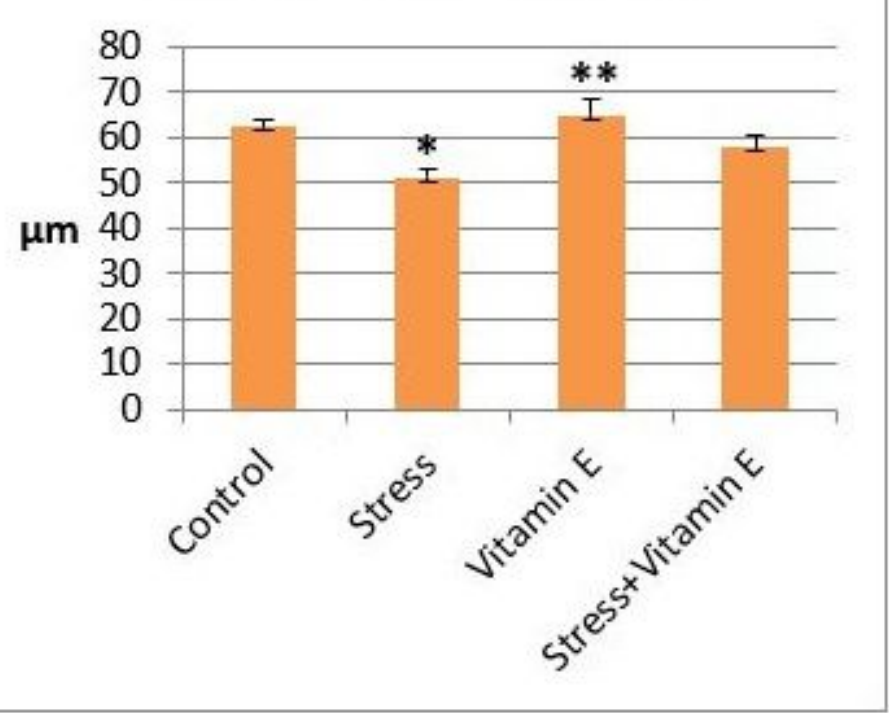

\section{Figure 6}

a) Adrenal capsule thickness of all groups. b) adrenal cortex thickness of all groups. ${ }^{*}$ control vs. stress group, ** vitamin E vs. stress group. 

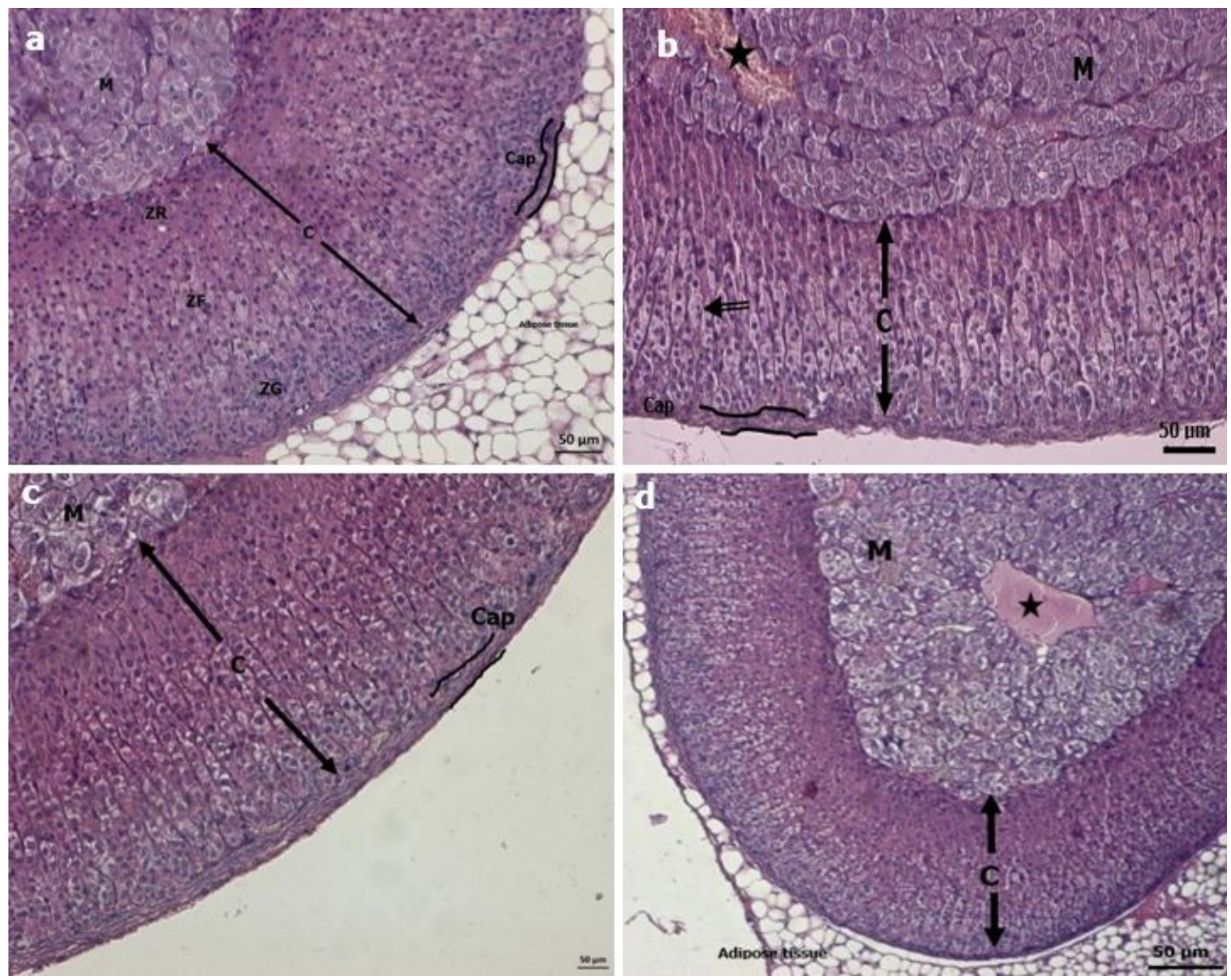

Figure 7

a) Normal histological view of the adrenal cortex and a medulla in the control group. Zona glomerulosa $(\mathrm{ZG})$, zona fasciculata (ZF), zona reticularis (ZR), medulla (M), cortex (C), and capsule (cap) with surrounding adipose tissue are seen. (H-E, Scale Bar: $50 \mu \mathrm{m})$. b) cortical atrophy, zona fasciculata with pyknotic nuclei (double-tailed arrow), and medullary hypertrophy with vascular dilatation (asterisk) in the stress group section. C: cortex, M: medulla, Cap: capsule. (H. E, Scale Bar: $50 \mu \mathrm{m})$. c) Adrenal section of the vitamin E group. The capsule (cap), adrenal cortex (C), and medulla (M) were seen in normal histological structure (he, Scale Bar: $50 \mu \mathrm{m}$ ). d) Cross-section of the adrenal gland with surrounding adipose tissue in the stress+vitamin $\mathrm{E}$ group. The gland seems to be histologically normal except for medullary vascular dilatation. C: cortex, M: medulla. (H. E, Scale Bar: $50 \mu \mathrm{m})$. 

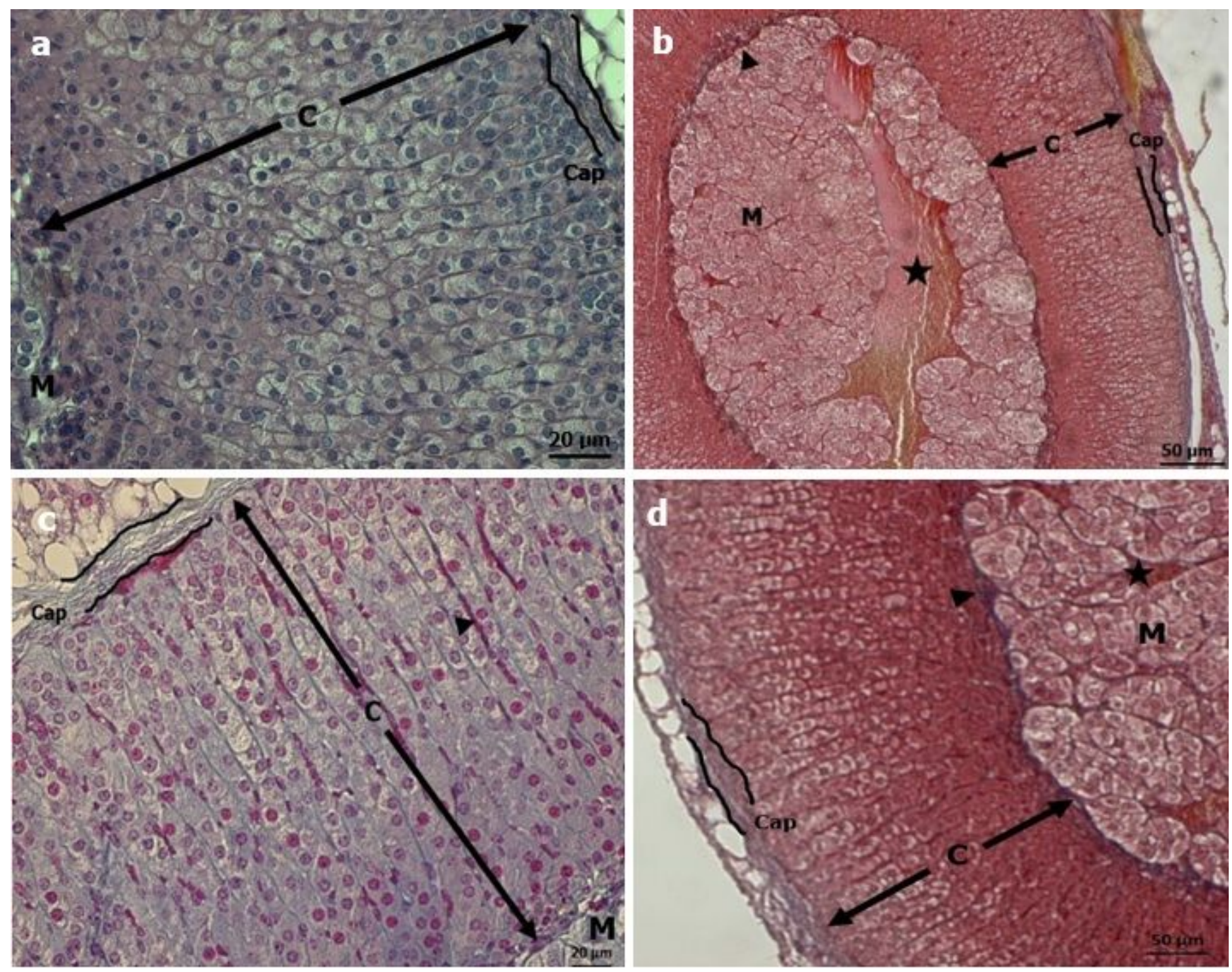

\section{Figure 8}

a) Azan-stained control group sections with capsule (cap), cortex (C), and partially medulla (M). (AZAN, Scale Bar: $20 \mu \mathrm{m}$ ). b) Increased interstitial fibrosis (arrowhead) in the corticomedullary border and hypertrophic medulla with vascular dilatation and hemorrhage (asterisk) in the stress group. C: cortex, M: medulla, Cap: capsule. (Azan, Scale Bar: $50 \mu \mathrm{m})$. c) Adrenal section of the vitamin E group. The normal histological appearance of capsule (Cap) cortex (C) with cortical sinusoid (arrowhead) and medulla (M) (Azan, Scale Bar: $20 \mu \mathrm{m}$ ). d) Normal histological view of an adrenal section with decreased interstitial fibrosis (arrowhead) in corticomedullary border and vascular dilatation (asterisk) in the medulla in the stress+vitamin E group. C: cortex, M: medulla, Cap: Capsule. (Azan, Scale Bar: $50 \mu \mathrm{m}$ ).

\section{Supplementary Files}

This is a list of supplementary files associated with this preprint. Click to download.

- allstatistics.xlsx 